\title{
High-Performance Polymer Solar Cells with Minimal Energy Loss Enabled by a
}

Main-Chain Twisting Nonfullerene Acceptor

Yan Zou, ${ }^{\dagger}$ Yingying Dong, ${ }^{\dagger}$ Chenkai Sun, ${ }^{\dagger}$ Yue Wu, ${ }^{\dagger}$ Hang Yang, ${ }^{\dagger}$ Chaohua Cui, ${ }^{*},{ }^{\dagger}$ and Yongfang $\mathrm{Li}^{\dagger}$;

'Laboratory of Advanced Optoelectronic Materials, College of Chemistry, Chemical Engineering and Materials Science \& Collaborative Innovation Center of Suzhou Nano Science and Technology, Soochow University, Suzhou 215123, China.

${ }^{*}$ Beijing National Laboratory for Molecular Sciences, CAS Key Laboratory of Organic Solids, Institute of Chemistry, Chinese Academy of Sciences, Beijing 100190, P. R. China.

E-mail: cuichaohua@suda.edu.cn

\section{Experimental Section}

\section{Materials}

5-bromo-4-((2-ethylhexyl)thio)thiophene-3-carbaldehyde (2)

Compound 1 (5 g, $16.3 \mathrm{mmol}$ ) in was dissolved in $40 \mathrm{~mL}$ anhydrous THF and stirred at $-78{ }^{\circ} \mathrm{C}$, then $8.5 \mathrm{~mL}(17 \mathrm{mmol}) \mathrm{LDA}$ was added dropwise. After stirring at that temperature for 1.5 hours, $2.76 \mathrm{~g}(24.4 \mathrm{mmol}) \mathrm{N}$-formylpiperidine was added in one portion and then the mixture was stirred overnight at room temperature for quenching the lithiation intermediate. Then the mixture was poured into ice water $(200 \mathrm{~mL})$ and then extracted with diethyl ether. The organic layer was washed with water three times and dried over $\mathrm{MgSO}_{4}$. After removal of the solvent, the crude product was purified by silica gel using petroleum ether as eluent to afford compound 2 as deep yellow oil. (1.47 g, yield 27\%)

${ }^{1} \mathrm{H}$ NMR (400 MHz, $\left.\mathrm{CDCl}_{3}\right) \delta$ (ppm): 9.95 (s, 1H), 7.08 (s, 1H), 2.98-2.96 (d, 2H), 1.57-0.88 (m, 15H). ${ }^{13} \mathrm{C}$ NMR (100 MHz, $\left.\mathrm{CDCl}_{3}\right) \delta(\mathrm{ppm}): 180.63,145.79,132.08$, 124.41, 39.21, 39.15, 32.14, 28.65, 25.41, 22.87, 14.03, 10.69. GC MS: calcd. for $\mathrm{C}_{13} \mathrm{H}_{19} \mathrm{BrOS}_{2} \mathrm{~m} / \mathrm{z}=335.32$; found 336.04 . 
5,5'-(4,4,9,9-tetrakis(4-hexylphenyl)-4,9-dihydro-s-indaceno[1,2-b:5,6-b']dithiophene -2,7-diyl)bis(4-((2-ethylhexyl)thio)thiophene-3-carbaldehyde)

Compound 2 (1.47 g, $4.4 \mathrm{mmol})$, compound 3 (2.16 g, $1.8 \mathrm{mmol})$ and $\mathrm{Pd}\left(\mathrm{PPh}_{3}\right)_{4}(253$ $\mathrm{mg}, 0.22 \mathrm{mmol}$ ) were charged into a flask with $40 \mathrm{ml}$ toluene under argon protection and stirred at $110^{\circ} \mathrm{C}$ overnight. After removing the solvent, the crude reaction mixture was directly subjected to column chromatography on silica gel using petroleum ether and dichloromethane as eluent $(1: 4, \mathrm{v} / \mathrm{v})$ to afford compound $4(1.8 \mathrm{~g}$, yield $72 \%)$ as red solid.

${ }^{1} \mathrm{H}$ NMR (400MHz, $\left.\mathrm{CDCl}_{3}\right): \delta$ (ppm): 10.00 (s, 2H), 7.42 (s, 2H), 7.26-7.24 (d, 4H), 7.16-7.14 (d, 8H), 7.10-7.08 (d, 8H), 3.00-2.99 (d, 4H), 2.59-2.55 (m, 8H), 1.57-0.87 $(\mathrm{m}, 74 \mathrm{H}) .{ }^{13} \mathrm{C}$ NMR $\left(100 \mathrm{MHz}, \mathrm{CDCl}_{3}\right): \delta(\mathrm{ppm}): 181.24,157.18,153.83,146.61$, $146.31,141.92,141.03,139.27,138.16,135.19,128.50,127.74,124.29,122.10$, 117.74, 114.04. MALDI-TOF MS: calcd. for $\mathrm{C}_{90} \mathrm{H}_{110} \mathrm{O}_{2} \mathrm{~S}_{6} \mathrm{~m} / z=1416.23$; found 1416.66 .

2,2'-((2Z,2 'Z)-(((4,4,9,9-tetrakis(4-hexylphenyl)-4,9-dihydro-s-indaceno[1,2-b:5,6-b'] dithiophene-2,7-diyl)bis(4-((2-ethylhexyl)thio)thiophene-5,3-diyl))bis(methanylyliden e))bis(5,6-difluoro-3-oxo-2,3-dihydro-1 H-indene-2,1-diylidene))dimalononitrile (IE4F-S)

2-(5,6-difluoro-3-oxo-2,3-dihydro-1H-inden-1-ylidene)malononitrile $(1.46$ g, 6.35 mmol) and Compound $4(1.8 \mathrm{~g}, 1.27 \mathrm{mmol})$ were charged into a flask with $100 \mathrm{~mL}$ $\mathrm{CHCl}_{3}$ under argon protection and stirred at $65{ }^{\circ} \mathrm{C}$ overnight. Then the reaction mixture was concentrated and precipitated into $30 \mathrm{ml}$ methanol. After filtration, the dark crude solid was washed with $50 \mathrm{ml}$ methanol and dried over vacuum drying. The crude solid was then subjected to column chromatography on silica gel using petroleum ether and chloromethane as eluent $(1: 1, \mathrm{v} / \mathrm{v})$ to afford IE4F-S $(2.1 \mathrm{~g}$, yield $90 \%)$ as dark solid.

${ }^{1} \mathrm{H}$ NMR (400 MHz, $\left.\mathrm{CDCl}_{3}\right): \delta(\mathrm{ppm}) 9.09$ (s, 2H), 8.55-8.51 (m, 2H), 7.65-7.62 (m, 2H), $7.48(\mathrm{~s}, 4 \mathrm{H}), 7.23(\mathrm{~s}, 2 \mathrm{H}), 7.18-7.16(\mathrm{~d}, 8 \mathrm{H}), 7.12-7.10(\mathrm{~d}, 8 \mathrm{H}), 3.09-3.08(\mathrm{~d}, 4 \mathrm{H})$, 2.60-2.57 (t, 8H), 1.57-0.87 (m, 69H). $\left.{ }^{13} \mathrm{C} \mathrm{NMR} \mathrm{(100} \mathrm{MHz,} \mathrm{CDCl}_{3}\right): \delta(\mathrm{ppm}): 186.28$, 
$158.60,158.35,158.15,154.52,153.35,153.04,152.38,145.50,142.12,140.67$, $138.41,136.58,135.58,134.32,133.98,129.88,128.61,127.74,123.86,119.79$, $118.13,114.75,114.35,112.39,112.26,69.09,63.17,39.87,39.24,35.55,32.29$, 31.69, 31.32, 29.08, 28.81, 25.55, 22.89, 22.56, 14.07, 10.82. MALDI-TOF MS: calcd. for $\mathrm{C}_{114} \mathrm{H}_{114} \mathrm{~F}_{4} \mathrm{~N}_{4} \mathrm{O}_{2} \mathrm{~S}_{6} m / z=1840.53$; found 1838.61 .

\section{Instruments and general methods}

A Cyclic voltammetry was performend on a Zahner IM6e electrochemical workstation with a three-electrode system in a solution of $0.1 \mathrm{M}\left[\mathrm{Bu}_{4} \mathrm{~N}\right] \mathrm{PF}_{6}$ acetonitrile solution at a scan rate of $100 \mathrm{mV} \mathrm{s}^{-1}$. Glassy carbon dis coated with polymer film was used as the working electrode. A Pt wire was used the counter electrode and $\mathrm{Ag} / \mathrm{Ag}^{+}$was used as the reference electrode. Ferrocene/ferrocenium redox couple was used as the external standard and its redox potential is $0.06 \mathrm{~V}$ versus $\mathrm{Ag} / \mathrm{Ag}^{+}$. The HOMO and LUMO energy levels were calculated from the onset of the oxidation and reduction potential of polymer using the following equations: $\mathrm{HOMO}=-\mathrm{e}\left(\varphi_{\mathrm{ox}}+4.74\right)(\mathrm{eV}) ; \mathrm{LUMO}=$ $-\mathrm{e}\left(\varphi_{\text {red }}+4.74\right)(\mathrm{eV})$, respectively.

\section{Solar cell fabrication and characterization}

The device was fabricated with a conventional architecture of ITO/PEDOT:PSS/active layer/PDINO/Al. The ITO-coated glass was pre-cleaned and modified by a thin layer of PEDOT:PSS which was spin-cast from a PEDOT:PSS aqueous solution (Baytron P VP Al 4083 from H. C. Stark) at $5000 \mathrm{rpm}$ for $45 \mathrm{~s}$, and then dried subsequently at $150{ }^{\circ} \mathrm{C}$ for $15 \mathrm{~min}$ in air. The thickness of the PEDOT:PSS layer is $\sim 30 \mathrm{~nm}$. Then the devices were transferred to a nitrogen-filled glovebox, where the active layer was spin-coated from chlorobenzene solution containing PBDB-T:IE4F-S (or PTQ10:IE4F-S) onto the PEDOT:PSS layer. The PBDB-T:IE4F-S (or PTQ10:IE4F-S) was dissolved in chlorobenzene with a concentration of $10 \mathrm{mg} / \mathrm{mL}$ (for polymer) and stirred under $80{ }^{\circ} \mathrm{C}$ for $12 \mathrm{~h}$. The top $100 \mathrm{~nm} \mathrm{Al} \mathrm{electrode} \mathrm{was} \mathrm{deposited} \mathrm{in} \mathrm{vacuum} \mathrm{onto} \mathrm{the} \mathrm{PDINO} \mathrm{layer} \mathrm{at} \mathrm{a} \mathrm{pressure} \mathrm{of}$ $\sim 5.0 \times 10^{-5} \mathrm{~Pa}$. The active area defined by a shadow mask is $2 \times 2 \mathrm{~mm}^{2}$. The current 
density-voltage $(J-V)$ measurement of the PSCs were measured under a illumination of AM 1.5G $\left(100 \mathrm{~mW} \mathrm{~cm}^{-2}\right)$ using a SS-F5-3A solar simulator (AAA grade, $50 \times 50$ $\mathrm{mm}^{2}$ photo beam size) of Enli Technology CO., Ltd.. The EQE was measured by using a Solar Cell Spectral Response Measurement System QE-R3011 (Enli Technology Co., Ltd.). The light intensity at each wavelength was calibrated by a standard single-crystal Si solar cell. The photovoltaic performance of PBDB-T:IE4F-S and PTQ10:IE4F-S based devices with various D/A ratios and different thermal annealing temperature are shown in Table S1 and Table S2, respectively.

The hole and electron mobilities were calculated by using the space-charge-limited current (SCLC) method.

$$
J \cong(9 / 8) \varepsilon \varepsilon_{0} \mu_{0} V^{2} \exp \left(0.89 \sqrt{V / E_{0} L}\right) / L^{3}
$$

Where $\varepsilon$ is the dielectric constant of the polymers, $\varepsilon_{0}$ is the permittivity of the vacuum, $\mu_{0}$ is the zero-field mobility, $E_{0}$ is the characteristic field, $J$ is the current density, and $L$ is the thickness of the film.

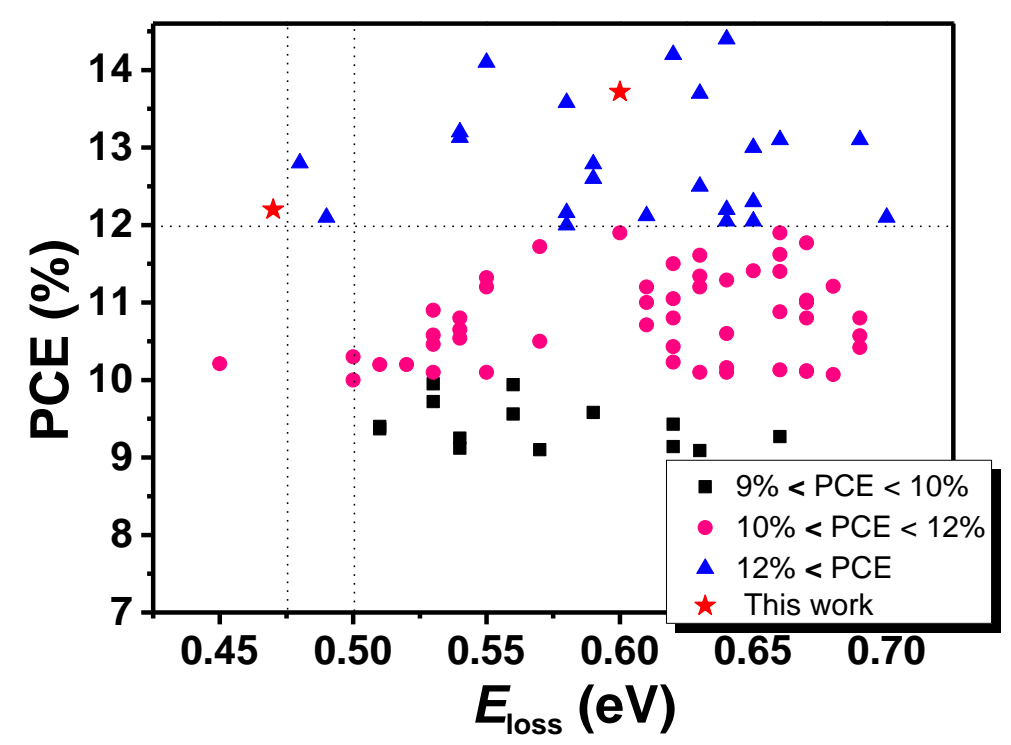

Figure S1. State-of-the-art $n$-OS based PSCs with efficiency over $9 \%$ and $E_{\text {loss }}$ below $0.7 \mathrm{eV}$ reported in literature. The key parameters are summarized in Table S4. 


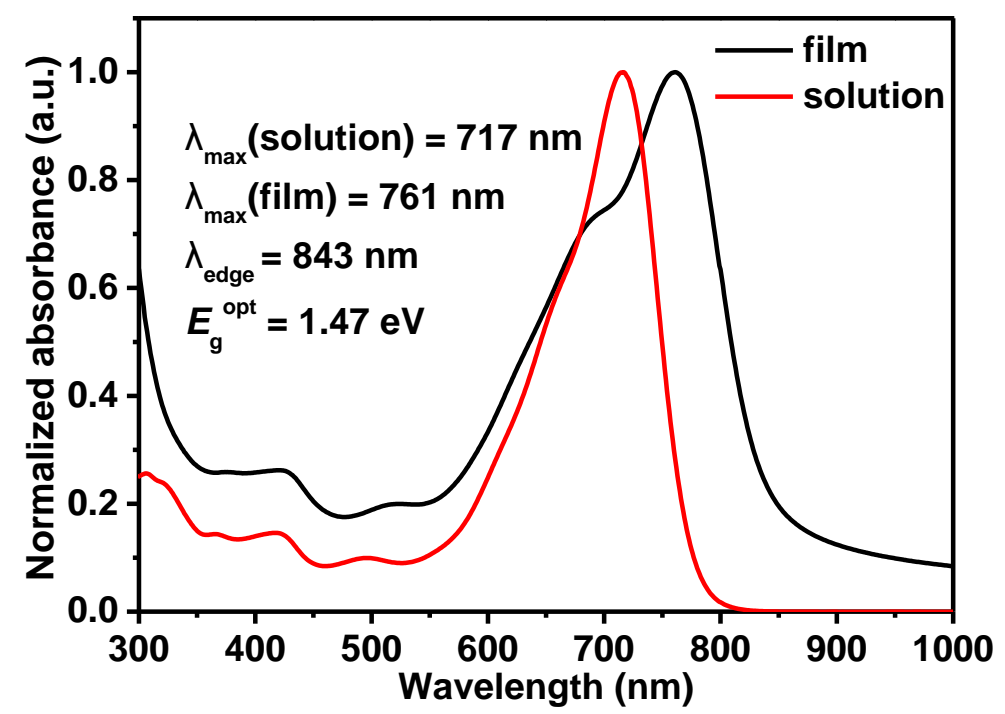

Figure S2. Normalized UV-vis Absorption spectra of IE4F-S in chloroform solution and solid thin film.

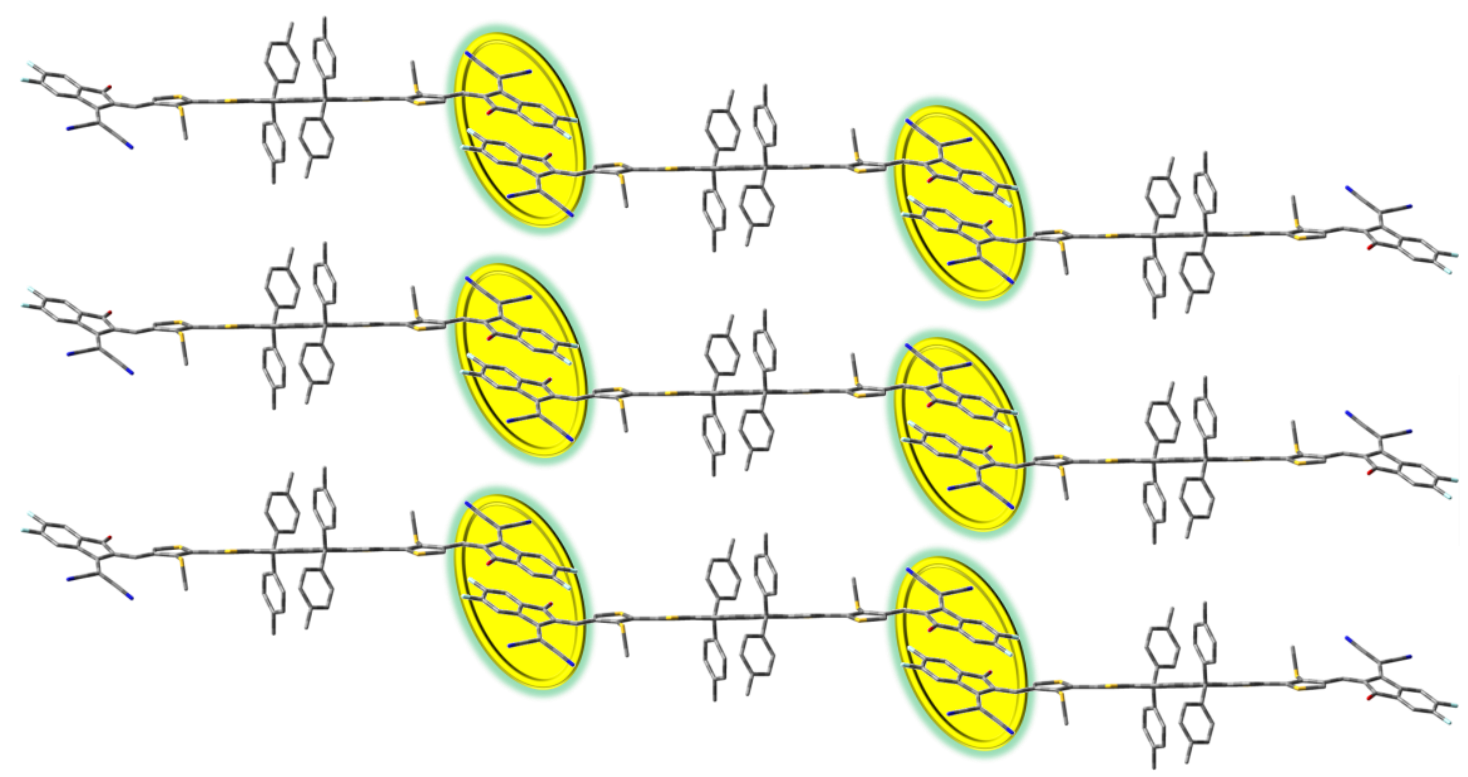

Figure S3. Simulated J-aggregates of IE4F-S neat films. 


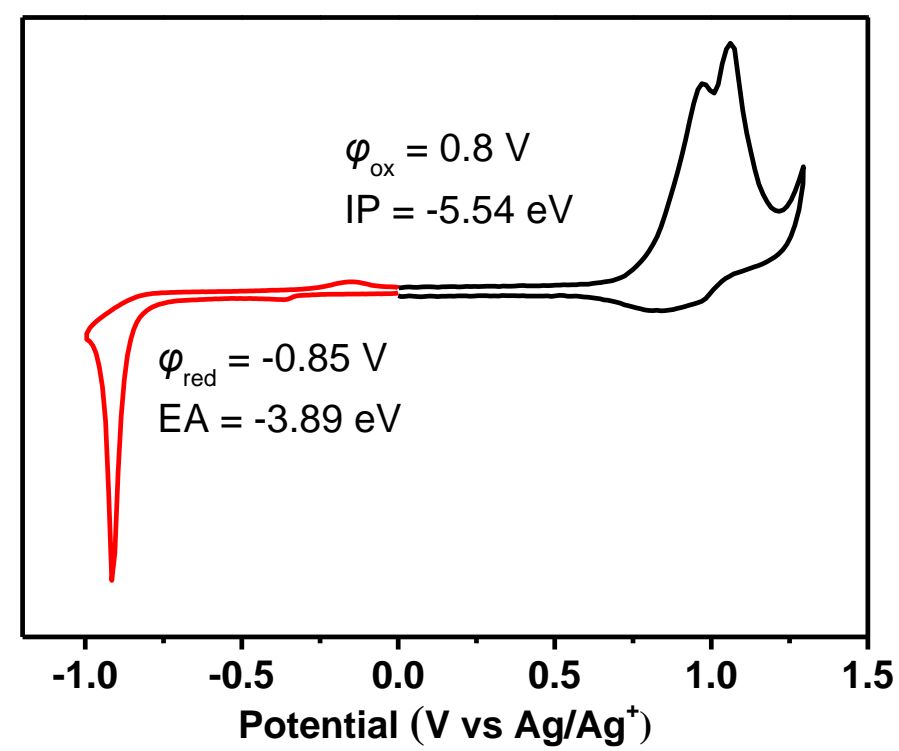

Figure S4. Cyclic voltammetry of IE4F-S film on a glass carbon electrode measured in $0.1 \mathrm{~mol} \mathrm{~L}^{-1} \mathrm{Bu}_{4} \mathrm{NPF}_{6}$ acetonitrile solution at a scan rate of $100 \mathrm{mV} \mathrm{s}^{-1}$.

(a)
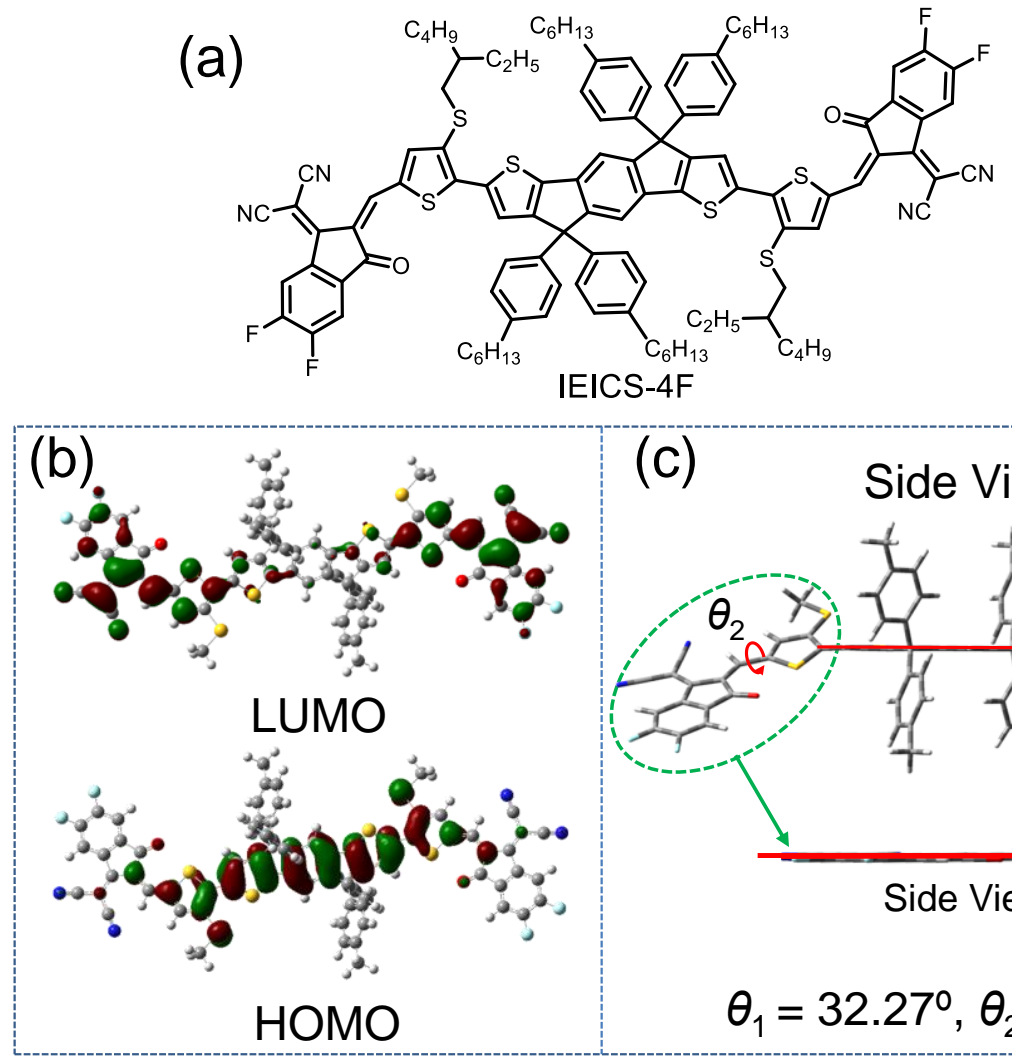

(c)

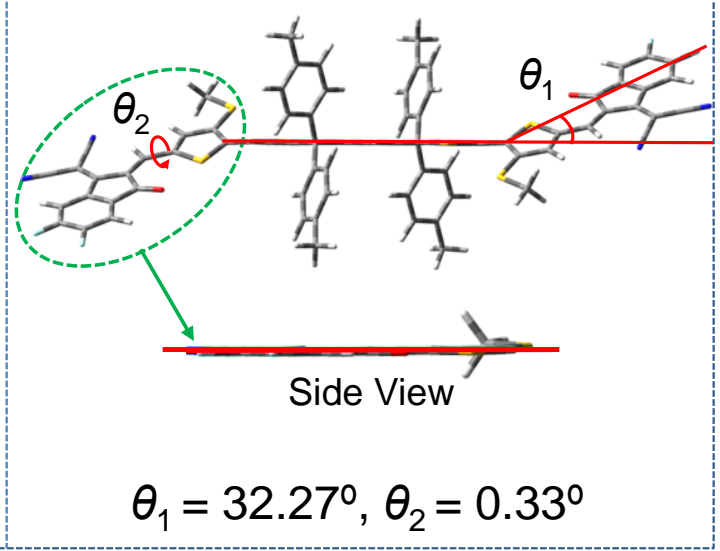

Figure S5. (a) Molecular structure of IEICS-4F, (b) Frontier molecular orbital surfaces and (c) optimized molecular conformations of IEICS-4F by DFT calculations at the $\omega \mathrm{B} 97 \mathrm{XD}$ functional and $6-31 \mathrm{G}^{* *}$ basis set with methyl groups in replacing alkyl substituents to simplify the calculations. 
Table S1. The photovoltaic performance of the PSCs with a device architecture of glass/ITO/PEDOT:PSS/PBDB-T:IE4F-S/PDINO/Al under the illumination of AM 1.5 $\mathrm{G}$ at $100 \mathrm{~mW} \mathrm{~cm}^{-2}$.

\begin{tabular}{cccccc}
\hline D/A ratio & TA [ $\left.{ }^{\circ} \mathrm{C}\right]$ & $V_{\text {oc }}[\mathrm{V}]$ & $J_{\text {sc }}\left[\mathrm{mAcm}^{-2}\right]$ & FF[\%] & PCE[\%] \\
\hline $1: 0.5$ & w/o & 0.880 & 20.76 & 55.2 & $10.06[9.96 \pm 0.10]$ \\
$1: 1$ & w/o & 0.879 & 21.69 & 60.1 & $11.46[11.31 \pm 0.17]$ \\
$1: 1.5$ & w/o & 0.860 & 22.58 & 51.5 & $10.05[10.01 \pm 0.06]$ \\
$1: 1$ & 100 & 0.877 & 20.99 & 69.4 & $12.80[12.62 \pm 0.15]$ \\
$1: 1$ & 120 & 0.869 & 21.95 & 68.9 & $13.11[12.91 \pm 0.16]$ \\
$1: 1$ & 140 & 0.877 & 22.30 & 68.0 & $13.29[13.16 \pm 0.10]$ \\
$1: 1$ & 160 & 0.868 & 22.88 & 69.1 & $13.72[13.48 \pm 0.19]$ \\
$1: 1$ & 180 & 0.843 & 21.60 & 70.4 & $12.83[12.62 \pm 0.17]$ \\
\hline
\end{tabular}

The statistical values in square bracket are the average PCE obtained from 6 devices.

Table S2. The photovoltaic performance of the PSCs with a device architecture of glass/ITO/PEDOT:PSS/PTQ10:IE4F-S/PDINO/Al under the illumination of AM 1.5

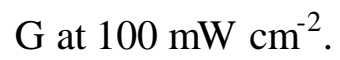

\begin{tabular}{cccccl}
\hline D/A ratio & TA [ $\left.{ }^{\circ} \mathrm{C}\right]$ & $V_{\mathrm{oc}}[\mathrm{V}]$ & $J_{\mathrm{sc}}\left[\mathrm{mAcm}^{-2}\right]$ & $\mathrm{FF}[\%]$ & PCE[\%] \\
\hline $1: 0.5$ & w/o & 1.002 & 16.31 & 40.5 & $6.62[6.43 \pm 0.10]$ \\
$1: 1$ & w/o & 0.986 & 18.19 & 57.2 & $10.26[10.16 \pm 0.07]$ \\
$1: 1.5$ & w/o & 0.993 & 19.29 & 58.2 & $11.14[11.04 \pm 0.14]$ \\
$1: 2$ & w/o & 0.997 & 14.51 & 55.1 & $7.97[7.87 \pm 0.11]$ \\
$1: 1.5$ & 100 & 0.991 & 19.54 & 60.7 & $11.75[11.49 \pm 0.18]$ \\
$1: 1.5$ & 120 & 0.986 & 20.25 & 60.4 & $12.05[11.92 \pm 0.16]$ \\
$1: 1.5$ & 140 & 0.996 & 19.67 & 62.3 & $12.20[11.98 \pm 0.14]$ \\
$1: 1.5$ & 160 & 0.984 & 18.99 & 62.6 & $11.69[11.47 \pm 0.19]$ \\
\hline
\end{tabular}

The statistical values in square bracket are the average PCE obtained from 6 devices. 
Table S3. Summary of the photovoltaicperformance of PBDB-T based single junction cells with PCE over $11 \%$.

\begin{tabular}{cccccc}
\hline Active layer & $V_{\text {oc }}[\mathrm{V}]$ & $J_{\text {sc }}\left[\mathrm{mA} \mathrm{cm}^{-2}\right]$ & FF[\%] & PCE[\%] & ref. \\
\hline PBDB-T:ITC6-IC & 0.970 & 16.41 & 73.0 & 11.61 & 1 \\
PBDB-T:6TIC-4F & 0.723 & 23.00 & 67.0 & 11.14 & 2 \\
PBDB-T:ITIC & 0.899 & 16.81 & 74.2 & 11.21 & 3 \\
PBDB-T:ITCC & 1.010 & 15.90 & 71.0 & 11.40 & 4 \\
PBDB-T:IDT6CN-M & 0.921 & 16.02 & 76.8 & 11.20 & 5 \\
PBDB-T:IT-M & 0.940 & 17.44 & 73.5 & 12.05 & 6 \\
PBDB-T:NITI & 0.860 & 20.67 & 71.0 & 12.74 & 7 \\
PBDB-T:NCBDT & 0.839 & 20.33 & 71.0 & 12.12 & 8 \\
PBDB-T:C8-ITIC & 0.870 & 19.70 & 73.0 & 12.41 & 9 \\
PBDB-T:IXIC-2Cl & 0.730 & 23.60 & 70.9 & 12.20 & 10 \\
PBDB-T:ITIC & 0.900 & 17.98 & 77.3 & 12.51 & 11 \\
PBDB-T:IT-M & 0.940 & 18.42 & 76.9 & 13.31 & 12 \\
PBDB-T:i-IEICO-4F:IDIC & 0.968 & 20.60 & 64.9 & 13.00 & 12 \\
PBDB-T:BTTIC & 0.904 & 19.52 & 74.7 & 13.18 & 13 \\
PBDB-T:ZITI & 0.890 & 19.80 & 74.0 & 13.03 & 14 \\
PBDB-T:IE4F-S & 0.868 & 22.88 & 69.1 & 13.72 & This \\
\hline
\end{tabular}
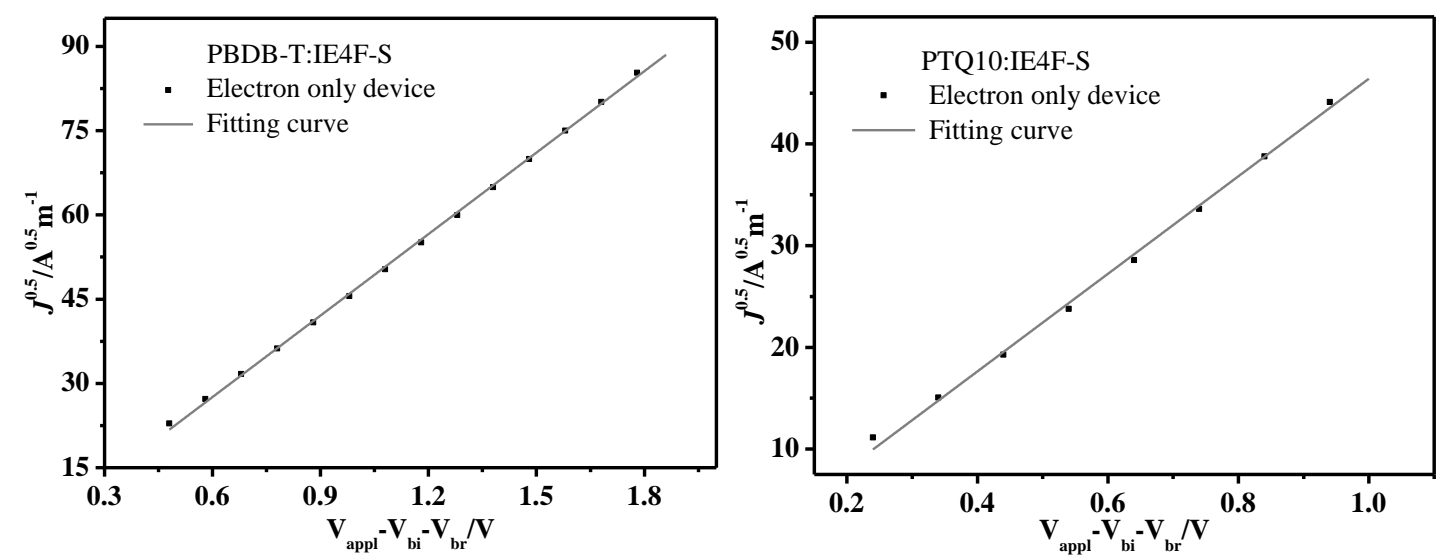

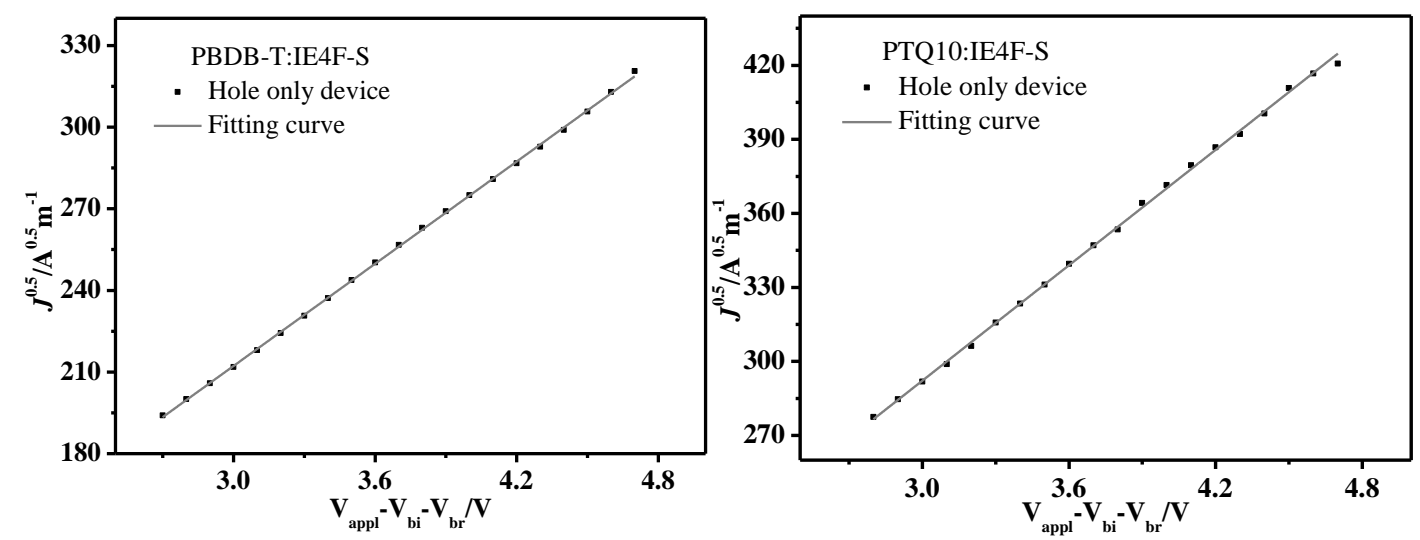

Figure S6. $J^{0.5} \mathrm{vs}\left(\mathrm{V}_{\mathrm{app}}-\mathrm{V}_{\mathrm{bi}}-\mathrm{V}_{\mathrm{br}}\right)$ plots hole-only and electron only devices of PBDB-T:IE4F-S (1:1, w/w) and PTQ10-T:IE4F-S (1:1.5, w/w) blend films, respectively.
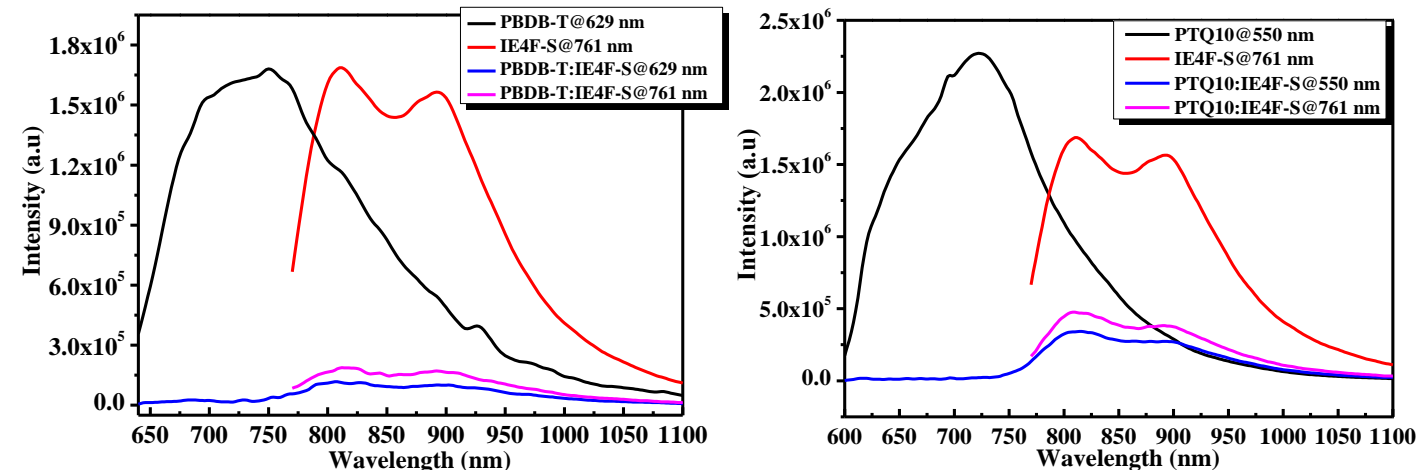

Figure S7. PL spectra of (a) PBDB-T, IE4F-S, and PBDB-T:IE4F-S blend film; (b) PTQ10, IE4F-S and PTQ10:IE4F-S blend film.

Table S4. Summary of the photovoltaic performance of single junction cells with PCE over $9 \%$ and $E_{\text {loss }}$ below $0.70 \mathrm{eV}$

\begin{tabular}{ccccccc}
\hline Active layer & $V_{\mathrm{oc}}[\mathrm{V}]$ & $J_{\mathrm{sc}}\left[\mathrm{mA} \mathrm{cm}^{-2}\right]$ & $\mathrm{FF}[\%]$ & PCE [\%] & $E_{\text {loss }}[\mathrm{eV}]$ & Ref. \\
\hline PBDB-T:NCBDT & 0.839 & 20.33 & 71.0 & 12.12 & 0.61 & 8 \\
J61:BT-IC & 0.870 & 16.35 & 67.1 & 9.56 & 0.56 & 15 \\
J71:BT-IC & 0.900 & 17.75 & 65.7 & 10.46 & 0.53 & 15 \\
FTAZ:IDCIC & 0.870 & 21.98 & 71.0 & 13.58 & 0.58 & 16 \\
PBDB-T:INPIC-4F & 0.850 & 21.61 & 71.5 & 13.13 & 0.54 & 17 \\
PTB7-Th:ATT-2 & 0.730 & 20.75 & 63.0 & 9.58 & 0.59 & 18 \\
PBDTTT-EFT:IEICO-4F & 0.739 & 22.80 & 59.4 & 10.00 & 0.50 & 19 \\
\hline
\end{tabular}




\begin{tabular}{|c|c|c|c|c|c|c|}
\hline J52:IEICO-4F & 0.734 & 21.90 & 58.5 & 9.40 & 0.51 & 19 \\
\hline PBDB-T:ITOIC-2Cl & 0.884 & 17.10 & 61.6 & 9.37 & 0.51 & 20 \\
\hline PBDB-T:IDTOT2F & 0.850 & 20.87 & 72.0 & 12.79 & 0.59 & 21 \\
\hline PBDB-T:IDT-EDOT & 0.860 & 21.34 & 62.0 & 11.32 & 0.55 & 22 \\
\hline J71:ITVfIC & 0.840 & 19.73 & 58.7 & 9.72 & 0.53 & 23 \\
\hline J71:ITVffIC & 0.810 & 20.60 & 63.2 & 10.54 & 0.54 & 23 \\
\hline PBDB-T1:ITTIC & 0.920 & 15.93 & 62.0 & 9.12 & 0.54 & 24 \\
\hline PTB7-Th:6TIC & 0.826 & 19.63 & 65.7 & 10.65 & 0.54 & 25 \\
\hline PTB7-Th:FOIC & 0.743 & 24.00 & 67.1 & 12.00 & 0.58 & 26 \\
\hline PTB7-Th:F8IC & 0.640 & 25.12 & 67.6 & 10.90 & 0.53 & 27 \\
\hline PTB7-Th:F10IC & 0.732 & 20.83 & 66.8 & 10.20 & 0.52 & 27 \\
\hline FTAZ:ITIC2 & 0.925 & 18.88 & 63.0 & 11.00 & 0.61 & 28 \\
\hline PFBTBOX:ITIC-4F & 1.040 & 15.35 & 65.0 & 10.20 & 0.51 & 29 \\
\hline CS01:PC ${ }_{71} \mathrm{BM}: M P U 3$ & 0.970 & 16.27 & 63.0 & 9.94 & 0.56 & 30 \\
\hline SMD: MPU3 & 0.980 & 13.72 & 67.0 & 9.14 & 0.62 & 31 \\
\hline PBT4Cl-Bz:IT-4F & 0.960 & 16.42 & 58.7 & 9.25 & 0.54 & 32 \\
\hline PTB7-Th:IDTT-T & 1.010 & 15.70 & 57.0 & 9.10 & 0.57 & 33 \\
\hline PBDB-T:SN6IC-4F & 0.780 & 23.20 & 73.0 & 13.20 & 0.54 & 34 \\
\hline PTB7-Th:DTPC-DFIC & 0.760 & 21.92 & 61.3 & 10.21 & 0.45 & 35 \\
\hline PBDB-T-SF:NCBDT-4Cl & 0.851 & 22.35 & 74.3 & 14.10 & 0.55 & 36 \\
\hline PTB7-Th:IOTIC-2F & 0.821 & 22.60 & 66.0 & 12.10 & 0.49 & 37 \\
\hline PTB7-Th:ITVfIC & 0.741 & 18.88 & 64.9 & 9.09 & 0.63 & 38 \\
\hline J71:ITIC5 & 0.897 & 18.48 & 75.5 & 12.50 & 0.63 & 39 \\
\hline J61:SJ-IC & 0.830 & 16.99 & 66.0 & 9.27 & 0.66 & 40 \\
\hline PBDB-T:IDT-BOC6 & 1.010 & 17.52 & 54.0 & 9.43 & 0.62 & 41 \\
\hline FTAZ:INIC1 & 0.929 & 16.63 & 64.3 & 10.10 & 0.63 & 42 \\
\hline PTB7-Th:4TIC & 0.780 & 18.80 & 72.0 & 10.43 & 0.62 & 43 \\
\hline FTAZ:INIC2 & 0.903 & 17.56 & 66.8 & 10.80 & 0.62 & 42 \\
\hline PBDB-T:FDNCTF & 0.909 & 15.90 & 74.6 & 10.80 & 0.69 & 44 \\
\hline
\end{tabular}




\begin{tabular}{|c|c|c|c|c|c|c|}
\hline PTB7-Th:IUIC & 0.796 & 21.74 & 64.9 & 11.20 & 0.61 & 45 \\
\hline PTB7-Th:BT-CIC & 0.700 & 22.50 & 71.0 & 11.20 & 0.63 & 46 \\
\hline FTAZ:F9IC:F11IC & 0.883 & 20.90 & 68.9 & 12.60 & 0.59 & 47 \\
\hline FTAZ:INIC3 & 0.857 & 19.44 & 67.4 & 11.50 & 0.62 & 42 \\
\hline PBDB-T:ITC6-IC & 0.970 & 16.49 & 73.0 & 11.61 & 0.63 & 1 \\
\hline FTAZ:ITIC2 & 0.925 & 18.88 & 63.0 & 11.00 & 0.61 & 28 \\
\hline FTAZ:IOIC2 & 0.900 & 19.70 & 69.3 & 12.30 & 0.65 & 48 \\
\hline PTB7-Th:FNIC2 & 0.741 & 23.93 & 73.4 & 13.00 & 0.65 & 49 \\
\hline PTB7-Th:IOIC3 & 0.762 & 22.90 & 74.9 & 13.10 & 0.69 & 50 \\
\hline PBDB-T:NFBDT-Me & 0.909 & 17.30 & 70.0 & 11.00 & 0.67 & 51 \\
\hline PBDB-T:NFBDT & 0.872 & 17.85 & 67.2 & 10.42 & 0.69 & 51 \\
\hline FTAZ:ITIC-Th1 & 0.849 & 19.33 & 73.7 & 12.10 & 0.70 & 52 \\
\hline P3TEA:FTTB-PDI4 & 1.130 & 13.89 & 65.9 & 10.58 & 0.53 & 53 \\
\hline PBDB-T:ITIC & 0.899 & 16.81 & 74.2 & 11.21 & 0.68 & 3 \\
\hline PBDB-T:IT-M & 0.940 & 17.44 & 73.5 & 12.05 & 0.65 & 6 \\
\hline PBDB-T:IT-DM & 0.970 & 16.48 & 70.6 & 11.29 & 0.64 & 6 \\
\hline J71:ITIC & 0.940 & 17.32 & 69.8 & 11.41 & 0.65 & 54 \\
\hline PTFB-O:ITIC & 0.920 & 15.50 & 70.0 & 10.13 & 0.66 & 55 \\
\hline PTFB-O:ITIC-Th & 0.920 & 17.10 & 67.0 & 10.88 & 0.66 & 55 \\
\hline PTFBDT-BZS:IDIC & 0.905 & 17.30 & 70.8 & 11.03 & 0.67 & 56 \\
\hline PDCBT:ITIC & 0.940 & 16.50 & 65.7 & 10.16 & 0.64 & 57 \\
\hline PBDB-T:ITCC & 1.010 & 15.90 & 71.0 & 11.40 & 0.66 & 4 \\
\hline J51:ITIC & 0.898 & 17.97 & 65.5 & 10.57 & 0.69 & 58 \\
\hline J51:m-ITIC & 0.912 & 18.31 & 70.6 & 11.77 & 0.67 & 58 \\
\hline J70:m-ITIC & 0.920 & 18.09 & 69.8 & 11.62 & 0.66 & 59 \\
\hline J71:m-ITIC & 0.944 & 18.09 & 70.6 & 12.05 & 0.64 & 59 \\
\hline J72:m-ITIC & 0.962 & 16.35 & 65.0 & 10.23 & 0.62 & 59 \\
\hline J73:m-ITIC & 0.974 & 16.45 & 66.9 & 10.71 & 0.61 & 59 \\
\hline J81:ITIC & 0.950 & 15.27 & 73.1 & 10.60 & 0.64 & 60 \\
\hline
\end{tabular}




\begin{tabular}{|c|c|c|c|c|c|c|}
\hline J81:m-IITIC & 0.960 & 16.48 & 69.8 & 11.05 & 0.62 & 60 \\
\hline SM1:IDIC & 0.905 & 15.18 & 73.6 & 10.11 & 0.67 & 61 \\
\hline FTAZ:INIC1 & 0.929 & 16.63 & 64.3 & 10.10 & 0.64 & 42 \\
\hline PBDB-T:IT-OM-2 & 0.930 & 17.53 & 73.0 & 11.90 & 0.66 & 62 \\
\hline PBDB-T:IT-OM-3 & 0.970 & 16.38 & 68.0 & 10.80 & 0.67 & 62 \\
\hline PBQ-4F:ITIC & 0.950 & 17.87 & 66.8 & 11.34 & 0.63 & 63 \\
\hline PBDB-TF:IDTN & 0.946 & 16.58 & 78.0 & 12.20 & 0.64 & 64 \\
\hline PB3T:IT-M & 1.000 & 18.90 & 63.0 & 11.90 & 0.60 & 65 \\
\hline J52:IEICO-4Cl & 0.700 & 23.80 & 60.7 & 10.10 & 0.53 & 66 \\
\hline PTB7-Th:IEICO-4Cl & 0.727 & 22.80 & 62.0 & 10.30 & 0.50 & 66 \\
\hline PBDB-T-SF:IT-4F & 0.880 & 20.50 & 71.9 & 13.10 & 0.66 & 67 \\
\hline PTB7-Th:ATT-1 & 0.870 & 16.48 & 70.0 & 10.07 & 0.68 & 68 \\
\hline PBPD-Th:ITIC & 1.010 & 18.10 & 59.0 & 10.80 & 0.54 & 69 \\
\hline PBDB-TF:IT-M & 1.020 & 15.78 & 65.0 & 10.50 & 0.57 & 70 \\
\hline PBDB-TF:IT-4F & 0.870 & 20.38 & 77.0 & 13.70 & 0.63 & 70 \\
\hline PBDB-T-2F:IT-4F & 0.860 & 21.80 & 77.0 & 14.40 & 0.64 & 71 \\
\hline PDTB-EF-T:IT-4F & 0.900 & 20.73 & 76.0 & 14.20 & 0.62 & 72 \\
\hline PTB7-Th:COi8DFIC & 0.680 & 26.12 & 68.2 & 12.16 & 0.58 & 73 \\
\hline PBDTS-TDZ:ITIC & 1.100 & 17.78 & 65.4 & 12.80 & 0.48 & 74 \\
\hline PBDT-TDZ:ITIC & 1.010 & 17.15 & 67.7 & 11.72 & 0.57 & 74 \\
\hline PffBT4T-2DT:IDTBR & 1.070 & 15.00 & 62.0 & 9.95 & 0.53 & 75 \\
\hline PTBF-Th:SFBRCN & 0.900 & 17.25 & 65.2 & 10.12 & 0.67 & 76 \\
\hline PvBDTTAZ:O-IDTBR & 1.080 & 16.26 & 63.6 & 11.20 & 0.55 & 77 \\
\hline PTB7-Th:IDT-2BR & 1.030 & 14.50 & 65.0 & 10.10 & 0.55 & 78 \\
\hline PBDB-T:IE4F-S & 0.868 & 22.88 & 69.1 & 13.72 & 0.60 & This work \\
\hline PTQ10:IE4F-S & 0.996 & 19.67 & 62.3 & 12.20 & 0.47 & This work \\
\hline
\end{tabular}

\section{References}


(1) Zhang, Z.; Yu, J.; Yin, X.; Hu, Z.; Jiang, Y.; Sun, J.; Zhou, J.; Zhang, F.; Russell, T. P.; Liu, F. Conformation Locking on Fused- Ring Electron Acceptor for High-Performance Nonfullerene Organic Solar Cells. Adv. Funct. Mater. 2018, 28, 1705095.

(2) Shi, X.; Liao, X.; Gao, K.; Zuo, L.; Chen, J.; Zhao, J.; Liu, F.; Chen, Y.; Jen, A. K. Y. An Electron Acceptor with Broad Visible-NIR Absorption and Unique Solid State Packing for As- Cast High Performance Binary Organic Solar Cells. Adv. Funct. Mater. 2018, 28, 1802324.

(3) Zhao, W.; Qian, D.; Zhang, S.; Li, S.; Inganäs, O.; Gao, F.; Hou, J. Fullerene- free poly mer solar cells with over $11 \%$ efficiency and excellent thermal stability. Adv. Mater. 2016, 28, 4734-4739.

(4) Yao, H.; Ye, L.; Hou, J.; Jang, B.; Han, G.; Cui, Y.; Su, G. M.; Wang, C.; Gao, B.; Yu, R. Achieving Highly Efficient Nonfullerene Organic Solar Cells with Improved Intermolecular Interaction and Open- Circuit Voltage. Adv. Mater. 2017,29, 1700254.

(5) Gao, W.; Zhang, M.; Liu, T.; Ming, R.; An, Q.; Wu, K.; Xie, D.; Luo, Z.; Zhong, C.; Liu, F. Asymmetrical Ladder- Type Donor- Induced Polar Small Molecule Acceptor to Promote Fill Factors Approaching 77\% for High- Performance Nonfullerene Polymer Solar Cells. Adv. Mater. 2018, 30, 1800052.

(6) Li, S.; Ye, L.; Zhao, W.; Zhang, S.; Mukherjee, S.; Ade, H.; Hou, J. Energy- level modulation of small- molecule electron acceptors to achieve over $12 \%$ efficiency in polymer solar cells. Adv. Mater. 2016, $28,9423-9429$.

(7) Xu, S. j.; Zhou, Z.; Liu, W.; Zhang, Z.; Liu, F.; Yan, H.; Zhu, X. A Twisted Thieno [3, 4- b] thiophene- Based Electron Acceptor Featuring a 14- $\pi$-Electron Indenoindene Core for High- Performance Organic Photovoltaics.Adv. Mater. 2017, 29, 1704510.

(8) Kan, B.; Zhang, J.; Liu, F.; Wan, X.; Li, C.; Ke, X.; Wang, Y.; Feng, H.; Zhang, Y.; Long, G. Fine- Tuning the Energy Levels of a Nonfullerene Small- Molecule Acceptor to Achieve a High Short- Circuit Current and a Power Conversion Efficiency over 12\% in Organic Solar Cells. Adv. Mater. 2018, 30, 1704904.

(9) Fei, Z.; Eisner, F. D.; Jiao, X.; Azzouzi, M.; Röhr, J. A.; Han, Y.; Shahid, M.; Chesman, A. S.; Easton, C. D.; McNeill, C. R. An Alkylated Indacenodithieno [3, 2-b] thiophene- Based Nonfullerene Acceptor with High Crystallinity Exhibiting Single Junction Solar Cell Efficiencies Greater than $13 \%$ with Low Voltage Losses. Adv. Mater. 2018, 30, 1705209.

(10) Chen, Y.; Liu, T.; Hu, H.; Ma, T.; Lai, J. Y. L.; Zhang, J.; Ade, H.; Yan, H. Modulation of End Groups for Low- Bandgap Nonfullerene Acceptors Enabling High- Performance Organic Solar Cells. Adv. Energy Mater. 2018, 8, 1801203.

(11) Nian, L.; Kan, Y.; Wang, H.; Gao, K.; Xu, B.; Rong, Q.; Wang, R.; Wang, J.; Liu, F.; Chen, J. Ternary non-fullerene polymer solar cells with $13.51 \%$ efficiency and a record-high fill factor of 78.13\%. Energy Environ. Sci. 2018, 11, 3392-3399.

(12) Xie, Y.; Huang, W.; Liang, Q.; Zhu, J.; Cong, Z.; Lin, F.; Yi, S.; Luo, G.; Yang, T.; Liu, S. High-Performance Fullerene-Free Polymer Solar Cells Featuring Efficient Photocurrent Generation from Dual Pathways and Low Nonradiative Recombination Loss. ACS Energy Lett. 2018, 4, 8-16.

(13) Gao, W.; Liu, T.; Ming, R.; Luo, Z.; Wu, K.; Zhang, L.; Xin, J.; Xie, D.; Zhang, G.; Ma, W. Near- Infrared Small Molecule Acceptor Enabled High- Performance Nonfullerene Polymer Solar Cells with Over 13\% Efficiency. Adv. Funct. Mater. 2018, 28, 1803128.

(14) Liu, W.; Zhang, J.; Zhou, Z.; Zhang, D.; Zhang, Y.; Xu, S.; Zhu, X. Design of a New Fused- Ring Electron Acceptor with Excellent Compatibility to W ide- Bandgap Poly mer Donors for High- Performance Organic Photovoltaics. Adv. Mater. 2018, 30, 1800403. 
(15) Li, Y.; Zhong, L.; Gautam, B.; Bin, H.-J.; Lin, J.-D.; Wu, F.-P.; Zhang, Z.; Jiang, Z.-Q.; Zhang, Z.-G.; Gundogdu, K.; Li, Y.; Liao, L.-S. A near-infrared non-fullerene electron acceptor for high performance polymer solar cells. Energy Environ. Sci. 2017, 10, 1610-1620.

(16) He, D.; Zhao, F.; Xin, J.; Rech, J. J.; Wei, Z.; Ma, W.; You, W.; Li, B.; Jiang, L.; Li, Y. A Fused Ring Electron Acceptor with Decacyclic Core Enables over 13.5\% Efficiency for Organic Solar Cells. Adv. Energy Mater. 2018, 8, 1802050.

(17) Sun, J.; Ma, X.; Zhang, Z.; Yu, J.; Zhou, J.; Yin, X.; Yang, L.; Geng, R.; Zhu, R.; Zhang, F. Dithieno [3, 2- $\left.b: 2^{\prime}, 3^{\prime}-d\right]$ pyrrol Fused Nonfullerene Acceptors Enabling Over 13\% Efficiency for Organic Solar Cells. Adv. Mater. 2018,30, 1707150.

(18) Liu, F.; Zhou, Z.; Zhang, C.; Zhang, J.; Hu, Q.; Vergote, T.; Liu, F.; Russell, T. P.; Zhu, X. Efficient Semitransparent Solar Cells with High NIR Responsiveness Enabled by a Small- Bandgap Electron Acceptor. Adv. Mater. 2017, 29, 1606574.

(19) Yao, H.; Cui, Y.; Yu, R.; Gao, B.; Zhang, H.; Hou, J. Design, Synthesis, and Photovoltaic Characterization of a Small Molecular Acceptor with an Ultra- Narrow Band Gap. Angew. Chem. Int. Ed. 2017, 56, 3045-3049.

(20) Feng, S.; Lu, H.; Liu, Z.; Liu, Y.; Li, C.; Bo, Z. Designing a High-Performance A-D-A Fused-Ring Electron Acceptor Noncovalently Conformational Locking and Tailoring Its End Groups. Acta Phys. -Chim. Sin. 2019, 35, 355-360.

(21) Liu, Y.; Li, M.; Zhou, X.; Jia, Q.-Q.; Feng, S.; Jiang, P.; Xu, X.; Ma, W.; Li, H.-B.; Bo, Z. Nonfullerene Acceptors with Enhanced Solubility and Ordered Packing for High-Efficiency Poly mer Solar Cells. ACS Energy Lett. 2018, 3, 1832-1839.

(22) Jiang, P.; Ming, S.; Jia, Q.-Q.; Liu, Y.; Lu, H.; Li, M.; Xu, X.; Li, H.-B.; Bo, Z. The influence of the $\pi$-bridging unit of fused-ring acceptors on the performance of organic solar cells. J. Mater. Chem. A 2018, 6, 21335-21340.

(23) Li, X.; Huang, H.; Bin, H.; Peng, Z.; Zhu, C.; Xue, L.; Zhang, Z.-G.; Zhang, Z.; Ade, H.; Li, Y. Synthesis and Photovoltaic Properties of a Series of Narrow Bandgap Organic Semiconductor Acceptors with Their Absorption Edge Reaching 900 nm. Chem. Mater. 2017, 29, 10130-10138.

(24) Zhang, Z.; Liu, W.; Rehman, T.; Ju, H.-X.; Mai, J.; Lu, X.; Shi, M.; Zhu, J.; Li, C.-Z.; Chen, H. Energy-level modulation of non-fullerene acceptors to achieve high-efficiency poly mer solar cells at a diminished energy offset. J. Mater. Chem. A 2017, 5, 9649-9654.

(25) Shi, X.; Chen, J.; Gao, K.; Zuo, L.; Yao, Z.; Liu, F.; Tang, J.; Jen, A. K. Y. Terthieno [3, 2- b] Thiophene (6T) Based Low Bandgap Fused- Ring Electron Acceptor for Highly Efficient Solar Cells with a High Short- Circuit Current Density and Low Open- Circuit Voltage Loss. Adv. Energy Mater. 2018, 8, 1702831.

(26) Li, T.; Dai, S.; Ke, Z.; Yang, L.; Wang, J.; Yan, C.; Ma, W.; Zhan, X. Fused Tris (thienothiophene)- Based Electron Acceptor with Strong Near-Infrared Absorption for High- Performance As- Cast Solar Cells. Adv. Mater. 2018,30, 1705969.

(27) Dai, S.; Li, T.; Wang, W.; Xiao, Y.; Lau, T. K.; Li, Z.; Liu, K.; Lu, X.; Zhan, X. Enhancing the Performance of Poly mer Solar Cells via Core Engineering of NIR-Absorbing Electron Acceptors. Adv. Mater. 2018, 30, 1706571.

(28) Wang, J.; Wang, W.; Wang, X.; Wu, Y.; Zhang, Q.; Yan, C.; Ma, W.; You, W.; Zhan, X. Enhancing Performance of Nonfullerene Acceptors via Side- Chain Conjugation Strategy. Adv. Mater. 2017, 29, 1702125. 
(29) Park, M.; Jung, J. W. An easily accessible low band gap polymer for efficient non-fullerene polymer solar cells with low Eloss of $0.55 \mathrm{eV}$. ACS Appl. Mater. Interfaces 2019, 11, 5435.

(30) Privado, M.; Seco, C. R.; Singhal, R.; Cruz, P. d. 1.; Langa, F.; Sharma, G. D.; Palomares, E. Reduced Energy Offsets and Low Energy Losses Lead to Efficient ( 10\% at 1 sun) Ternary Organic Solar Cells. ACS Energy Lett. 2018, 3, 2418-2424.

(31) Privado, M.; de la Cruz, P.; Biswas, S.; Singhal, R.; Sharma, G. D.; Langa, F. A non-fullerene all s mall molecule solar cell constructed with a diketopyrrolopyrrole-based acceptor having a power conversion efficiency higher than $9 \%$ and an energy loss of $0.54 \mathrm{eV}$. J. Mater. Chem. A 2018, 6, 11714-11724.

(32) Chao, P.; Liu, L.; Zhou, J.; Qu, J.; Mo, D.; Meng, H.; Xie, Z.; He, F.; Ma, Y. Multichloro-Substitution Strategy: Facing Low Photon Energy Loss in Nonfullerene Solar Cells. ACS Appl. Energy Mater. 2018, 1, 6549-6559.

(33) He, B.; Yang, B.; Kolaczkowski, M. A.; Anderson, C. A.; Klivansky, L. M.; Chen, T. L.; Brady, M. A.; Liu, Y. Molecular engineering for large open-circu it voltage and low energy loss in around 10\% non-fullerene organic photovoltaics. ACS Energy Lett. 2018, 3, 1028-1035.

(34) Huang, C.; Liao, X.; Gao, K.; Zuo, L.; Lin, F.; Shi, X.; Li, C.-Z.; Liu, H.; Li, X.; Liu, F. Highly efficient organic solar cells based on S, N-heteroacene non-fullerene acceptors. Chem. Mater. 2018, 30, 5429-5434.

(35) Yao, Z.; Liao, X.; Gao, K.; Lin, F.; Xu, X.; Shi, X.; Zuo, L.; Liu, F.; Chen, Y.; Jen, A. K.-Y. Dithienopicenocarbazole-based acceptors for efficient organic solar cells with optoelectronic response over $1000 \mathrm{~nm}$ and an extremely low energy loss. J. Am. Chem. Soc. 2018, 140, 2054-2057.

(36) Kan, B.; Feng, H.; Yao, H.; Chang, M.; Wan, X.; Li, C.; Hou, J.; Chen, Y. A chlorinated low-bandgap small-molecule acceptor for organic solar cells with $14.1 \%$ efficiency and low energy loss. Sci. China Chem. 2018,61, 1307-1313.

(37) Lee, J.; Ko, S. J.; Seifrid, M.; Lee, H.; McDowell, C.; Luginbuhl, B. R.; Karki, A.; Cho, K.; Nguyen, T. Q.; Bazan, G. C. Design of Nonfullerene Acceptors with Near- Infrared Light Absorption Capabilities. Adv. Energy Mater. 2018, 8, 1801209.

(38) Huang, H.; Li, X.; Zhong, L.; Qiu, B.; Yang, Y.; Zhang, Z.-G.; Zhang, Z.; Li, Y. High performance as-cast semitransparent polymer solar cells. J. Mater. Chem. A 2018, 6, 4670-4677.

(39) Yan, C.; Wang, W.; Lau, T.-K.; Li, K.; Wang, J.; Liu, K.; Lu, X.; Zhan, X. Enhancing the performance of non-fullerene organic solar cells via end group engineering of fused-ring electron acceptors. J. Mater. Chem. A 2018, 6, 16638-16644.

(40) Li, X.; Yan, T.; Bin, H.; Han, G.; Xue, L.; Liu, F.; Yi, Y.; Zhang, Z.-G.; Russell, T. P.; Li, Y. Insertion of double bond $\pi$-bridges of A-D-A acceptors for high performance near-infrared poly mer solar cells. J. Mater. Chem. A 2017, 5, 22588-22597.

(41) Liu, Y.; Zhang, Z.; Feng, S.; Li, M.; Wu, L.; Hou, R.; Xu, X.; Chen, X.; Bo, Z. Exploiting noncovalently conformational locking as a design strategy for high performance fused-ring electron acceptor used in polymer solar cells. J. Am. Chem. Soc. 2017, 139, 3356-3359.

(42) Dai, S.; Zhao, F.; Zhang, Q.; Lau, T.-K.; Li, T.; Liu, K.; Ling, Q.; Wang, C.; Lu, X.; You, W.; Zhan, X. Fused Nonacyclic Electron Acceptors for Efficient Polymer Solar Cells. J. Am. Chem. Soc. 2017, 139, 1336-1343.

(43) Shi, X.; Zuo, L.; Jo, S. B.; Gao, K.; Lin, F.; Liu, F.; Jen, A. K.-Y. Design of a highly crystalline low-band gap fused-ring electron acceptor for high-efficiency solar cells with low energy loss. Chem. Mater. 2017, 29, 8369-8376. 
(44) Kan, B.; Yi, Y. Q. Q.; Wan, X.; Feng, H.; Ke, X.; Wang, Y.; Li, C.; Chen, Y. Ternary organic solar cells with $12.8 \%$ efficiency using two nonfullerene acceptors with complementary absorptions. Adv. Energy Mater. 2018, 8, 1800424.

(45) Jia, B.; Dai, S.; Ke, Z.; Yan, C.; Ma, W.; Zhan, X. Breaking 10\% efficiency in semitransparent solar cells with fused-undecacyclic electron acceptor. Chem. Mater. 2017, 30, 239-245.

(46) Li, Y.; Lin, J.-D.; Che, X.; Qu, Y.; Liu, F.; Liao, L.-S.; Forrest, S. R. High efficiency near-infrared and semitransparent non-fullerene acceptor organic photovoltaic cells. J. Am. Chem. Soc. 2017, 139, 17114-17119.

(47) Dai, S.; Xiao, Y.; Xue, P.; James Rech, J.; Liu, K.; Li, Z.; Lu, X.; You, W.; Zhan, X. Effect of Core Size on Performance of Fused-Ring Electron Acceptors. Chem. Mater. 2018, 30, 5390-5396.

(48) Zhu, J.; Ke, Z.; Zhang, Q.; Wang, J.; Dai, S.; Wu, Y.; Xu, Y.; Lin, Y.; Ma, W.; You, W. Naphthodithiophene- Based Nonfullerene Acceptor for High- Performance Organic Photovoltaics: Effect of Extended Conjugation. Adv. Mater. 2018, 30, 1704713.

(49) Wang, J.; Zhang, J.; Xiao, Y.; Xiao, T.; Zhu, R.; Yan, C.; Fu, Y.; Lu, G.; Lu, X.; Marder, S. R.; Zhan, X. Effect of Isomerization on High-Performance Nonfullerene Electron Acceptors. J. Am. Chem. Soc. 2018, 140, 9140-9147.

(50) Zhu, J.; Xiao, Y.; Wang, J.; Liu, K.; Jiang, H.; Lin, Y.; Lu, X.; Zhan, X. Alkoxy-Induced Near-Infrared Sensitive Electron Acceptor for High-Performance Organic Solar Cells. Chem. Mater. 2018, 30, 4150-4156.

(51) Ke, X.; Kan, B.; Wan, X.; Wang, Y.; Zhang, Y.; Li, C.; Chen, Y. Substituents on the end group subtle tuning the energy levels and absorptions of small-molecule nonfullerene acceptors. Dyes Pigments 2018, 155, 241-248.

(52) Zhao, F.; Dai, S.; Wu, Y.; Zhang, Q.; Wang, J.; Jiang, L.; Ling, Q.; Wei, Z.; Ma, W.; You, W. Single- Junction Binary- Blend Nonfullerene Poly mer Solar Cells with $12.1 \%$ Efficiency. Adv. Mater. 2017, 29, 1700144.

(53) Zhang, J.; Li, Y.; Huang, J.; Hu, H.; Zhang, G.; Ma, T.; Chow, P. C.; Ade, H.; Pan, D.; Yan, H. Ring-fusion of pery lene diimide acceptor enabling efficient nonfullerene organic solar cells with a small voltage loss.J. Am. Chem. Soc. 2017, 139, 16092-16095.

(54) Bin, H.; Gao, L.; Zhang, Z.-G.; Yang, Y.; Zhang, Y.; Zhang, C.; Chen, S.; Xue, L.; Yang, C.; Xiao, M. $11.4 \%$ Efficiency non-fullerene polymer solar cells with trialkylsilyl substituted 2D-conjugated polymer as donor. Nature Commun. 2016, 7, 13651.

(55) Li, Z.; Jiang, K.; Yang, G.; Lai, J. Y. L.; Ma, T.; Zhao, J.; Ma, W.; Yan, H. Donor poly mer design enables efficient non-fullerene organic solar cells. Nature Commun. 2016, 7, 13094.

(56) Lin, Y.; Zhao, F.; Wu, Y.; Chen, K.; Xia, Y.; Li, G.; Prasad, S. K.; Zhu, J.; Huo, L.; Bin, H. Mapping Polymer Donors toward High- Efficiency Fullerene Free Organic Solar Cells. Adv. Mater. 2017, 29, 1604155.

(57) Qin, Y.; Uddin, M. A.; Chen, Y.; Jang, B.; Zhao, K.; Zheng, Z.; Yu, R.; Shin, T. J.; Woo, H. Y.; Hou, J. Highly Efficient Fullerene- Free Polymer Solar Cells Fabricated with Polythiophene Derivative. Adv. Mater. 2016, 28, 9416-9422.

(58) Yang, Y.; Zhang, Z.-G.; Bin, H.; Chen, S.; Gao, L.; Xue, L.; Yang, C.; Li, Y. Side-chain isomerization on an n-type organic semiconductor ITIC acceptor makes $11.77 \%$ high efficiency polymer solar cells. J. Am. Chem. Soc. 2016, 138, 15011-15018. 
(59) Bin, H.; Yang, Y.; Peng, Z.; Ye, L.; Yao, J.; Zhong, L.; Sun, C.; Gao, L.; Huang, H.; Li, X. Effect of Alkylsily 1 Side- Chain Structure on Photovoltaic Properties of Conjugated Poly mer Donors. Adv. Energy Mater. 2018, 8, 1702324.

(60) Bin, H.; Zhong, L.; Yang, Y.; Gao, L.; Huang, H.; Sun, C.; Li, X.; Xue, L.; Zhang, Z. G.; Zhang, Z. Medium Bandgap Polymer Donor Based on Bi (trialkylsilylthienyl- benzo [1, 2- b: 4, 5- b']- difuran) for High Performance Nonfullerene Poly mer Solar Cells. Adv. Energy Mater. 2017, 7, 1700746 .

(61) Qiu, B.; Xue, L.; Yang, Y.; Bin, H.; Zhang, Y.; Zhang, C.; Xiao, M.; Park, K.; Morrison, W.; Zhang, Z.-G. All-s mall-molecule nonfullerene organic solar cells with high fill factor and high efficiency over 10\%. Chem. Mater. 2017, 29, 7543-7553.

(62) Li, S.; Ye, L.; Zhao, W.; Zhang, S.; Ade, H.; Hou, J. Significant Influence of the Methoxyl Substitution Position on Optoelectronic Properties and Molecular Packing of Small- Molecule Electron Acceptors for Photovoltaic Cells. Adv. Energy Mater. 2017, 7, 1700183.

(63) Zheng, Z.; Awartani, O. M.; Gautam, B.; Liu, D.; Qin, Y.; Li, W.; Bataller, A.; Gundogdu, K.; Ade, H.; Hou, J. Efficient charge transfer and fine-tuned energy level alignment in a THF- processed fullerene- free organic solar cell with $11.3 \%$ efficiency. Adv. Mater. 2017, 29, 1604241.

(64) Li, S.; Ye, L.; Zhao, W.; Liu, X.; Zhu, J.; Ade, H.; Hou, J. Design of a New Small- Molecule Electron Acceptor Enables Efficient Poly mer Solar Cells with High Fill Factor. Adv. Mater. 2017, 29, 1704051.

(65) Liu, D.; Yang, B.; Jang, B.; Xu, B.; Zhang, S.; He, C.; Woo, H. Y.; Hou, J. Molecular design of a wide-band-gap conjugated polymer for efficient fullerene-free poly mer solar cells. Energy Environ. Sci. 2017, 10, 546-551.

(66) Cui, Y.; Yang, C.; Yao, H.; Zhu, J.; Wang, Y.; Jia, G.; Gao, F.; Hou, J. Efficient Semitransparent Organic Solar Cells with Tunable Color enabled by an Ultralow-Bandgap Nonfullerene Acceptor. Adv. Mater. 2017, 29, 1703080.

(67) Zhao, W.; Li, S.; Yao, H.; Zhang, S.; Zhang, Y.; Yang, B.; Hou, J. Molecular optimization enables over $13 \%$ efficiency in organic solar cells. J. Am. Chem. Soc. 2017, 139, 7148-7151.

(68) Liu, F.; Zhou, Z.; Zhang, C.; Vergote, T.; Fan, H.; Liu, F.; Zhu, X. A thieno [3, 4-b] thiophene-based non-fullerene electron acceptor for high-performance bulk-heterojunction organic solar cells. J. Am. Chem. Soc. 2016, 138, 15523-15526.

(69) Fan, Q.; Xu, Z.; Guo, X.; Meng, X.; Li, W.; Su, W.; Ou, X.; Ma, W.; Zhang, M.; Li, Y. High-performance nonfullerene poly mer solar cells with open-circuit voltage over $1 \mathrm{~V}$ and energy loss as low as 0.54 eV. Nano Energy 2017, 40, 20-26.

(70) Li, W.; Ye, L.; Li, S.; Yao, H.; Ade, H.; Hou, J. A High- Efficiency Organic Solar Cell Enabled by the Strong Intramolecular Electron Push-Pull Effect of the Nonfullerene Acceptor. Adv. Mater. 2018, 30, 1707170.

(71) Zhang, S.; Qin, Y.; Zhu, J.; Hou, J. Over $14 \%$ efficiency in polymer solar cells enabled by a chlorinated polymer donor. Adv. Mater. 2018, 30, 1800868.

(72) Li, S.; Ye, L.; Zhao, W.; Yan, H.; Yang, B.; Liu, D.; Li, W.; Ade, H.; Hou, J. A wide band gap polymer with a deep highest occupied molecular orbital level enables $14.2 \%$ efficiency in polymer solar cells. J. Am. Chem. Soc. 2018, 140, 7159-7167. 
(73) Xiao, Z.; Jia, X.; Li, D.; Wang, S.; Geng, X.; Liu, F.; Chen, J.; Yang, S.; Russell, T. P.; Ding, L. 26 mA c m-2 Jsc from organic solar cells with a low-bandgap nonfullerene acceptor. Sci. Bull. 2017, 62, 1494-1496.

(74) Xu, X.; Yu, T.; Bi, Z.; Ma, W.; Li, Y.; Peng, Q. Realizing over $13 \%$ efficiency in green- solvent- processed nonfullerene organic solar cells enabled by 1, 3, 4- thiadiazole- based wide- bandgap copolymers. Adv. Mater. 2018, 30, 1703973.

(75) Baran, D.; Kirchartz, T.; Wheeler, S.; Dimitrov, S.; Abdelsamie, M.; Gorman, J.; Ashraf, R.; Holliday, S.; Wadsworth, A.; Gasparini, N. Reduced voltage losses yield $10 \%$ efficient fullerene free organic solar cells with> $1 \mathrm{~V}$ open circuit voltages. Energy Environ. Sci. 2016, 9, 3783-3793.

(76) Zhang, G.; Yang, G.; Yan, H.; Kim, J. H.; Ade, H.; Wu, W.; Xu, X.; Duan, Y.; Peng, Q. Efficient nonfullerene polymer solar cells enabled by a novel wide bandgap small molecular acceptor. Adv. Mater. 2017,29, 1606054.

(77) Chen, S.; Liu, Y.; Zhang, L.; Chow, P. C.; Wang, Z.; Zhang, G.; Ma, W.; Yan, H. A wide-bandgap donor polymer for highly efficient non-fullerene organic solar cells with a s mall voltage loss.J. Am. Chem. Soc. 2017, 139, 6298-6301.

(78) Cheng, P.; Zhang, M.; Lau, T. K.; Wu, Y.; Jia, B.; Wang, J.; Yan, C.; Qin, M.; Lu, X.; Zhan, X. Realizing small energy loss of $0.55 \mathrm{ev}$, high open- circuit voltage $>1 \mathrm{~V}$ and high efficiency> $10 \%$ in fullerene- free polymer solar cells via energy driver. Adv. Mater. 2017, 29, 1605216. 